\section{Measuring and reporting disease progression in subclinical rheumatic heart disease}

\author{
Ganesan Karthikeyan
}

Echocardiography is a sensitive test for detecting subclinical rheumatic heart disease (RHD) in asymptomatic individuals. However, it also detects a large number of minor abnormalities which may overlap with the normal range of variability in valve morphology and function in the population. These abnormalities do not fulfil criteria for the diagnosis of RHD and have been variously termed 'possible' or 'borderline' by different guidelines. ${ }^{12}$ The utility of routine screening echocardiography in endemic populations hinges on two critical parameters: (1) the rate of disease progression and (2) the impact of secondary antibiotic prophylaxis in stemming this progression. Data on disease progression from the earliest echocardiographic screening studies are now becoming available. But, these studies are generally limited by the small number of cases, short follow-up times, substantial attrition rates, and in the studies which preceded the World Heart Federation (WHF) recommendations, inconsistent diagnostic criteria. $^{13-8}$

In this issue, Engelman and colleagues present findings from a retrospective study on disease progression in a screening-detected population in Fiji. The authors used the WHF criteria for diagnosis and report outcomes at a median of 7.5 years, which is the longest duration of follow-up reported till date. Four of seventeen people with borderline RHD progressed to definite RHD, and four of twenty with definite RHD went on to require valve replacement. Based on these data, the authors conclude that screeningdetected valve disease is not benign. Is this assertion valid? Is it generalisable to other populations with subclinical RHD? Are these findings consistent with prior data? This study presents us with an opportunity to review and reassess the measurement and reporting of disease progression in studies of subclinical RHD.

Department of Cardiology, All India Institute of Medical Sciences, New Delhi, India

Correspondence to Professor Ganesan Karthikeyan, Department of Cardiology, Room 24, Cardiothoracic Sciences Centre, All India Institute of Medical Sciences, New Delhi 110029, India; karthik2010@gmail.com (RHD) progression to valve replacement. RHD.

\section{MEASURING DISEASE PROGRESSION}

Studies have used the term progression to imply either a change in classification from borderline to definite RHD or, less frequently, worsening of the severity of valve lesions in definite RHD. Often, these have been clubbed together as a single, composite measure of progression of subclinical disease. Reclassification from borderline to definite RHD may result from (a) development of pathological regurgitation on a morphologically abnormal valve, (b) occurrence of morphological changes in a previously unaffected valve or (c) occurrence of significant mitral stenosis. Therefore, the clinical implications of progression by differing mechanisms may be different, but these details are seldom reported. The reporting of progression in definite RHD is even less consistent. Some studies have reported progression of definite lesions when the severity of regurgitation increased by at least one grade. ${ }^{3}$ However, it is reasonable to assume that disease has progressed if a previously unaffected valve develops morphological or functional changes, or if significant mitral stenosis develops. ${ }^{6}$ Classification of these changes as 'stable' or 'persistent' disease entails a significant loss of information regarding disease progression. The annualised rates of progression reported in the published literature are summarised in table 1 . This summary highlights the gaps in reporting and the variability in the rates of progression seen among studies using different criteria.

\section{MEASURING DETERMINANTS OF PROGRESSION}

There are few adequately powered studies which have identified potential predictors of disease progression. Most studies have reported univariate associations of baseline variables with disease progression or persistence (often combining the two as a single outcome). ${ }^{8}$ Morphological characteristics of the valve at the time of detection have been shown to be associated with disease progression, ${ }^{7}$ and overcrowding with persistence or progression. ${ }^{6}$ Use of secondary prophylaxis has not been shown to be protective in the published studies and was paradoxically, independently associated with progression in one study. ${ }^{7}$ One critical variable which has not been adequately evaluated as a predictor of progression in screening studies is the severity of regurgitation at baseline. In the study by Engelman et al, the majority of patients with definite RHD (12/20) appeared to have had moderate (nine patients) or severe disease (three patients) at baseline, four of these progressing to have valve replacement surgery. These data are consistent with the 24-month results of the Rheumatic Heart Disease Global Registry (REMEDY). In this registry, severity of valve disease was the strongest independent predictor of disease progression related clinical outcomes (such as death and heart failure). ${ }^{9}$

\section{GENERALISABILITY OF RESULTS}

Screening studies have been performed in populations across countries and communities at different stages in the evolution of RHD epidemiology, and burden of

Table 1 Annualised rates of progression of borderline and definite rheumatic heart disease

\begin{tabular}{lcl}
\hline Study (year) & $\begin{array}{l}\text { Progression of borderline to } \\
\text { definite RHD (\% per year) }\end{array}$ & $\begin{array}{l}\text { Progression of definite } \\
\text { RHD (\% per year)* }\end{array}$ \\
\hline Paar et al $(2010)^{1} \dagger$ & 21.1 & - \\
Saxena et al $(2011)^{3}$ & - & 3.2 \\
Bhaya et al $(2011)^{4}$ & - & 1.3 \\
Beaton et al $(2014)^{5}$ & 4.7 & - \\
Mirabel et al $(2015)^{6}$ & - & 4.3 \\
Rémond et al $(2015)^{7}$ & 7.2 & - \\
Zühlke et al $(2016)^{8} 9$ & 4.6 & - \\
Engelman et al $(2016)$ & 3.6 & 3.0 \\
\hline
\end{tabular}

* Rate of progression of regurgitation by one grade of severity except for the study by Engelman, which reports

tThis study did not use the World Heart Federation (WHF) criteria; progression is from possible or probable to definite 
disease. And, as such, generalising rates of progression derived from one community to another is fraught. As an example, are the progression rates reported by Engelman and colleagues applicable to a country like India, where RHD is perhaps on the decline? One approach to address this question would be to assess the baseline risk of the populations for comparability. Progression of subclinical disease is presumably related to recurrences of acute rheumatic fever (ARF). Since ARF incidence is rarely available for populations, surrogate measures of disease transmission in unselected populations that could be used are the prevalence of definite and borderline RHD. In cohorts with significant loss to follow-up, the ratio of definite to borderline RHD and the proportion of patients with moderate or severe disease could also provide an indication of representativeness. There were more definite RHD cases than borderline RHD cases (20 vs 17) in the study by Engelman. Typically, borderline RHD is three to five times more frequent than definite RHD in screening studies. ${ }^{5} 9$ This suggests that the cohort reported by Engelman may be an 'enriched' population, the result of selection bias due to differential follow-up of patients with borderline and definite RHD. Risk of progression at baseline may also be related to the proportion of patients with moderate or severe disease at the time of screening, which is perhaps a reflection of the health-seeking behaviour of the population. The large proportion of patients with moderate or severe disease $(12 / 20,60 \%)$ in this study, as against the $10 \%$ typically found in screening studies, ${ }^{3}$ also predicates a higher than usual risk of progression.

\section{FUTURE STUDIES}

The first step in evaluating the utility of screening echocardiography for detecting subclinical RHD is to obtain valid estimates of the rate and determinants of disease progression. It is only with this knowledge that we can test the effectiveness of interventions to retard progression. The principal requirement for any such study is that it needs to be sufficiently large. If the annual rate of progression of subclinical RHD is assumed to be $3 \%$, in order to test the usefulness of seven to ten potential predictors of progression, 700-1000 patients with subclinical RHD would need to be followed up for at least 5 years, with very low attrition rates. In order to understand the full clinical impact of subclinical RHD, follow-up times need to be over 5 years. In addition,

Box 1 Key requirements for studies evaluating progression of subclinical rheumatic heart disease

Sample size

- A minimum of 700-1000 patients with subclinical rheumatic heart disease (RHD), followed up for at least 5 years, with minimal loss to follow-up

Diagnosis of subclinical disease

- Standardised protocol and trained personnel

- Consistent diagnostic criteria for diagnosis and classification

- Blinded assessment of echocardiographic images

- Report interobserver variability between readers

Baseline risk assessment

- Sociodemographic variables

- Prevalence of definite and borderline RHD; ratio of definite to borderline RHD

- Proportion of patients with moderate or severe valve disease at baseline

Diagnosis of disease progression

- Consistent diagnostic criteria for progression of definite RHD

- Report rates of progression of borderline and definite RHD separately

- Report rates of progression of definite RHD separate from persistent disease

- Report specific reasons for diagnosing progression (eg, worsening of regurgitation, new disease in an unaffected valve, etc)

Assessment of predictors of progression

- Report independent predictors of progression based on multivariable analyses

these studies need to incorporate several important elements into their design, conduct and reporting (box 1). Definitive data on progression will likely take international collaborative efforts involving multidisciplinary teams. ${ }^{10}$ In the meantime, investigators keen to do screening studies should be encouraged to harmonise their study protocols with others in the field and prospectively plan pooled analysis of data.

\section{Competing interests None declared.}

Provenance and peer review Commissioned; internally peer reviewed.

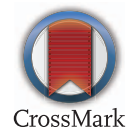

To cite Karthikeyan G. Heart Asia 2016;8:74-75. doi:10.1136/heartasia-2016-010857

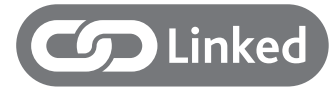

http://dx.doi.org/10.1136/heartasia-2016-010847

Heart Asia 2016;8:74-75.

doi:10.1136/heartasia-2016-010857

\section{REFERENCES}

1 Paar JA, Berrios NM, Rose JD, et al. Prevalence of rheumatic heart disease in children and young adults in Nicaragua. Am J Cardiol 2010;105:1809-14.

2 Reményi B, Wilson N, Steer A, et al. World Heart Federation criteria for echocardiographic diagnosis of rheumatic heart disease-an evidence-based guideline. Nat Rev Cardiol 2012;9:297-309.
3 Saxena A, Ramakrishnan S, Roy A, et al. Prevalence and outcome of subclinical rheumatic heart disease in India: the RHEUMATIC (Rheumatic Heart Echo Utilisation and Monitoring Actuarial Trends in Indian Children) study. Heart 2011;97:2018-22.

4 Bhaya M, Beniwal R, Panwar S, et al. Two years of follow-up validates the echocardiographic criteria for the diagnosis and screening of rheumatic heart disease in asymptomatic populations. Echocardiography 2011;28:929-33.

5 Beaton A, Okello E, Aliku T, et al. Latent rheumatic heart disease: outcomes 2 years after echocardiographic detection. Pediatr Cardiol 2014;35:1259-67.

6 Mirabel M, Fauchier T, Bacquelin R, et al. Echocardiography screening to detect rheumatic heart disease: a cohort study of schoolchildren in French Pacific Islands. Int J Cardiol 2015;188:89-95.

7 Rémond $\mathrm{M}$, Atkinson $\mathrm{D}$, White $\mathrm{A}$, et al. Are minor echocardiographic changes associated with an increased risk of acute rheumatic fever or progression to rheumatic heart disease? Int I Cardiol 2015;198:117-22.

8 Zühlke L, Engel ME, Lemmer $C E$, et al. The natural history of latent rheumatic heart disease in a 5 year follow-up study: a prospective observational study. BMC Cardiovasc Disord 2016;16:46.

9 Zühlke L, Karthikeyan G, Engel ME, et al. Clinical outcomes in 3343 children and adults with rheumatic heart disease from 14 low and middle income countries: 2-year follow-up of the global rheumatic heart disease registry (the REMEDY study). Circulation 2016;134:1456-66.

10 Sanyahumbi AS, Aliku T, Carapetis JR, et al. PT288 the concept and design of Definerhd: a study to evaluate the progression of subclinical rheumatic valve lesions diagnosed through echocardiographic screening. Global Heart 2016;11:e174-e5. 